Manuelle Medizin 2009 · 47:136-136

DOI 10.1007/s00337-009-0674-1

Online publiziert: 5. April 2009

๑) Springer Medizin Verlag 2009

\author{
F.C. Hundhausen \\ Kassel
}

\title{
Morbus Menière - ein skeletales Syndrom?
}

\section{Wer hat ähnliche Befunde gesehen?}

Laut Lehrbuch ist die Ursache der „Menière-Trias" nicht bekannt. Der Chirotherapeut denkt unmittelbar an die vegetative Beziehung von Wirbelblockierungen der Halswirbelsäule zur Kopfregion. Und hier wird er fündig: Die HWS zeigt eine ausgeprägte (skoliotische) Seitausbiegung, eventuell mit einer Kyphose. Dazu findet sich eine fortlaufende Blockierung mindestens der Wirbelkörper C1$C_{3}$, alle in gleicher Richtung. Segmentzugehörige Symptome sind Drehschwindel, Erbrechen und Taubheit des Ohres. Wird anamnestisch ein Halbseitenkopfschmerz auf der Skolioseseite angegeben, ist das Kopfgelenk Okziput/C1 zusätzlich blockiert. Das Zentrum scheint beim labilen HWK 3 zu liegen.

Hier sollte auch die Therapie beginnen. Der HWK 3 spricht am sichersten auf eine Manipulation der Kiefergelenke über dem Unterkiefer an. Die Wirbel werden per Impuls und Dehnung mobilisiert.

Theoretisch scheint das alles ganz einfach, aber da steht noch die pathologische Statik der Skoliose und/oder eine Kyphose der Therapie als hartnäckige Crux im Wege.

Entsprechend langwierig wird der Therapieverlauf. Bei länger bestehender Symptomatik erzielt man möglicherweise nur eine - allerdings eindeutige - Besserung des Leidens bezüglich Häufigkeit und Heftigkeit der Anfälle.

Ich kann meine Aussage leider nur auf zwei Fälle stützen, wobei die eine Patientin (35 Jahre) als Morbus Menière (vermutlich zur Neuraltherapie) überwiesen wurde.
Diesen Fall sowie die entsprechenden Röntgenbilder konnte ich über zwei Jahre beobachten.

Eine andere Patientin konnte ich nur manuell untersuchen. Die Beschwerden bestanden seit etwa einem Jahr. Der Befund war jedoch (beeindruckend) eindeutig. Es wurde eine Therapie mit zwei Impulsen und Dehnung (PIR) durchgeführt. Eine Stunde später untersuchte ein anderer - erfahrener - Chirotherapeut die Patientin. Er fand eine fast normale Halswirbelsäule vor.

Theoretisch muss hier eine „Schnappverspannung" vorgelegen haben. Dafür spricht die kurze Anamnese und das jugendliche Alter. Leider konnte ich mich vom Ergebnis nicht mehr überzeugen.

Ursache des Morbus Menière kann eine ausgeprägte pathologische Anlage der Wirbelsäule sein. Man sollte aber auch an den chronischen Bauchschläfer denken, der langzeitig den Kopf immer auf der gleichen Seite ablegt. Hier wäre eine konsequente Änderung der Schlafhaltung der erste Therapieschritt.

Wer hat ähnliche Befunde gesehen?

\section{Korrespondenzadresse \\ Dr. F.C. Hundhausen}

Im Weidengarten 7, 34130 Kassel

mac@drhundhausen.de 